\title{
First snapshot on behavioral characteristics and related factors of patients with chronic kidney disease in South Korea during the COVID-19 pandemic (June to October 2020)
}

\author{
Yaerim Kim${ }^{1}$, Inae Lee ${ }^{2}$, Jeonghwan Lee ${ }^{3}$, Jae Yoon Park ${ }^{4}$, Jung Nam An ${ }^{5}$, Kyung Don Yoo ${ }^{6}$, Yong Chul Kim, \\ Woo Yeong Park ${ }^{1}$, Kyubok Jin ${ }^{1}$, Younglim Kho ${ }^{8}$, Myoungsoon You ${ }^{2}$, Dong Ki Kim ${ }^{7}$, Kyungho Choi ${ }^{2}$, Jung Pyo Lee ${ }^{3}$ \\ ${ }^{1}$ Department of Internal Medicine, Keimyung University School of Medicine, Daegu, Republic of Korea \\ ${ }^{2}$ Department of Environmental Health Sciences, School of Public Health, Seoul National University, Seoul, Republic of Korea \\ ${ }^{3}$ Department of Internal Medicine, SMG-SNU Boramae Medical Center, Seoul, Republic of Korea \\ ${ }^{4}$ Department of Internal Medicine, Dongguk University Ilsan Hospital, Goyang, Republic of Korea \\ ${ }^{5}$ Department of Internal Medicine, Hallym University Sacred Heart Hospital, Anyang, Republic of Korea \\ ${ }^{6}$ Department of Internal Medicine, Ulsan University Hospital, University of Ulsan College of Medicine, Ulsan, Republic of Korea \\ ${ }^{7}$ Department of Internal Medicine, Seoul National University Hospital, Seoul, Republic of Korea \\ ${ }^{8}$ Department of Health, Environment, and Safety, Eulji University, Seongnam, Republic of Korea
}

Background: The recent novel coronavirus disease 2019 (COVID-19) pandemic has led to unprecedented changes in behavior. We evaluated the current status of precautionary behavior and physical activity in chronic kidney disease (CKD) patients during the COVID-19 pandemic.

Methods: A population of CKD patients $(n=306)$ registered in the Study on Kidney Disease and Environmental Chemicals (SKETCH, Clinical Trial No. NCT04679168) cohort recruited from June 2020 to October 2020 was included in the study. We conducted a questionnaire survey related to risk perception of COVID-19, precautionary behavior, and physical activity.

Results: There were 187 patients (61.1\%) with estimated glomerular filtration rate of $<45 \mathrm{~mL} / \mathrm{min} / 1.73 \mathrm{~m}^{2}$. This population showed a higher degree of risk perception for COVID-19 than the general population. Age was the most significant determinant of risk perception among CKD patients. During the pandemic, social distancing and hygiene-related behavior were significantly increased ( $p<$ 0.001). The frequency of exercise was decreased only in those who took regular exercise, without diabetes, or with a lower Charlson comorbidity index $(\mathrm{CCl})(\mathrm{p}<0.001)$, with no change among the other groups. Socioeconomic status and comorbidities significantly af-

Received: March 16, 2021; Revised: August 23, 2021; Accepted: August 23, 2021

Correspondence: Kyungho Choi

Department of Environmental Health Sciences, School of Public Health, Seoul National University, 1 Gwanak-ro, Gwanak-gu, Seoul 08826, Republic of Korea. E-mail: kyungho@snu.ac.kr

ORCID: https://orcid.org/0000-0001-7460-792X

Jung Pyo Lee

Department of Internal Medicine, SMG-SNU Boramae Medical Center, Seoul National University College of Medicine, 20 Boramae-ro 5-gil,

Dongjak-gu, Seoul 07061, Republic of Korea. E-mail: nephrolee@gmail.com

ORCID: https://orcid.org/0000-0002-4714-1260

Yaerim Kim and Inae Lee contributed equally to this work as co-first authors.

Copyright (C) 2022 by The Korean Society of Nephrology

(a) This is an Open Access article distributed under the terms of the Creative Commons Attribution Non-Commercial and No Derivatives License (http:// creativecommons.org/licenses/by-nc-nd/4.0/) which permits unrestricted non-commercial use, distribution of the material without any modifications, and reproduction in any medium, provided the original works properly cited. 
fected behavioral characteristics regardless of the category. Education and income were significantly associated with precautionary behaviors such as staying at home and hand sanitizer use. Patients with higher $\mathrm{CCl}$ status significantly increased frequency of exercise (adjusted odds ratio, 2.10; 95\% confidence interval, 1.01-4.38).

Conclusion: CKD patients showed higher risk perception with active precautionary behavioral changes than the general population. Healthcare providers should be aware of the characteristics to comprise precautionary behavior without reducing physical activity.

Keywords: Chronic kidney disease, COVID-19, Exercise, Health behavior

\section{Introduction}

The severe acute respiratory syndrome coronavirus 2 or coronavirus disease 2019 (COVID-19) pandemic has caused unprecedented threats to public health, disproportionally against the old and those with underlying health conditions. As the incidence rate of COVID-19 has increased exponentially, social distancing and the use of personal protective equipment (PPE) have been recommended and often enforced worldwide. Social distancing measures range from strict nationwide lockdown and/or curfew to more voluntary-based recommendations. In countries such as South Korea, varying stages of social (or physical) distancing measures were implemented depending on the extent of the viral spread in combination with rapid screening and efficient contact tracing without imposing draconian 'stay-at-home' restrictions [1-3]. Regardless of the extent of legal enforcement, the common factors in social distancing include avoidance of contact with other people, hygienic behavior, and the use of PPE.

Chronic kidney disease (CKD) is a common health problem worldwide, with a global prevalence reaching $29.3 \%$ in 2017 [4]. CKD is not only recognized as a risk factor for infectious diseases but may also aggravate their prognosis [5-7]. Indeed, CKD patients exhibit a significantly higher fatality rate following COVID-19 infection than the general population [8]. It is necessary to protect CKD patients from potential COVID-19 infection. Hence, health practitioners and clinicians need to understand the current status of compliance with precautionary recommendations among CKD patients, and the factors that influence their precautionary behaviors.

While active social distancing measures have contributed to a decrease in new cases by reducing the likelihood of transmitting COVID-19, these measures may lead to undesirable consequences, including physical inactivity.
Recent initial reports indicate that the stay-at-home orders in several countries caused significant decreases in physical activity not only among general or healthy populations but also among patients who require routine physical exercise $[9,10]$. Physical inactivity is responsible for approximately 3.2 million deaths per year [11]. Moreover, for CKD patients, a lack of physical activity has often been linked to adverse prognoses [12-14]. For this reason, we evaluate the impact of behavioral change on the physical activity of CKD patients.

This study was conducted to determine the current status of precautionary behaviors against the COVID-19 pandemic among CKD patients along with their physical activity during the first months of the pandemic. The observations made in this study can help to develop the most appropriate clinical recommendations in this vulnerable population to prevent COVID-19 infection and to promote healthy behavior that will improve the maintenance of underlying disease.

\section{Methods}

\section{Study populations}

This study was conducted with a cohort of CKD patients. The Study on Kidney Disease and Environmental Chemicals (SKETCH, Clinical Trial No. NCT04679168) aims to investigate the behavioral characteristics, chemical exposure, and clinical outcomes of CKD patients during and after the COVID-19 pandemic. We defined CKD as (1) estimated glomerular filtration rate (eGFR) of $\geq 15$ and $<60$ $\mathrm{mL} / \mathrm{min} / 1.73 \mathrm{~m}^{2}$ or (2) eGFR of $\geq 60 \mathrm{~mL} / \mathrm{min} / 1.73 \mathrm{~m}^{2}$ and urine protein to creatinine ratio of $>0.3 \mathrm{~g} / \mathrm{g}$. A total of 308 participants were recruited from five university hospitals located in Seoul, Ilsan, and Daegu, Korea between June and October 2020 (Supplementary Fig. 1, available online). 
Exclusion criteria included those who were followed up less than 3 months or with a recent history of acute kidney injury, progressive malignancy, cerebral infarction, cerebral hemorrhage, myocardial infarction, under hemodialysis, or immunosuppressant use.

The study protocol and consent to participate were approved by the Institutional Review Board in participating hospitals (Supplementary data 1, available online). The study was conducted in accordance with the principles of the Declaration of Helsinki.

\section{Questionnaire and clinical data}

A questionnaire survey was conducted by trained surveyors for participating patients on their visit to the hospital. The questionnaire included questions related to 1) risk perception of COVID-19, 2) hygienic behavior, 3) social distancing, and 4) physical activity during the past year (before the pandemic) and the 1 month before the hospital visit (during the pandemic). Detailed information of the survey was described in Supplementary data 2 (available online). Perceived risk of COVID-19 infection was determined by a 5-point scale from 'never' (1) to 'extremely high' (5); items, marked 4 or 5 were grouped as high-risk perception or 'high perceived risk.' For evaluating the risk perception among the general population of Korea, the relevant data (i.e., the possibility of personal COVID-19 infection) was gleaned from Hankook Research (https:// hrcopinion.co.kr/covid-19, accessed December 18, 2020) which collected data from 1,000 subjects through biweekly survey events. The statistics for the incidence of COVID-19 infection were obtained from the Korean Statistical Information Service (https://kosis.kr/ covid/covid_index.do, accessed December 13, 2020).

Demographic data, anthropometric data, and clinical laboratory data related to CKD were obtained when the subject visited the hospital (Supplementary data 3, available online). Data on underlying comorbidities were collected via electronic medical records using prescription records and diagnostic codes according to the International Classification of Diseases 10th Revision (ICD-10).

\section{Statistical analysis}

The chi-square tests and Fisher exact tests for categorical variables were conducted to compare demographic and clinical parameters by CKD status. In addition, the Mann-Whitney U test and the Student $\mathrm{t}$ test were performed for continuous variables. Categorical variables were expressed as numerical with proportions (\%), and continuous variables were expressed as mean \pm standard deviation when distributed normally (as median with interquartile range otherwise).

To compare behaviors before and during the COVID-19 pandemic, the Wilcoxon signed-rank test was used. Logistic regression analysis was conducted to identify the relative factors related to risk recognition or behavior changes. Sensitivity analysis was conducted with age adjustment because the odds ratio (OR) of high-degree perception was greater than scale ' 3 ' in the groups aged $\geq 60$ years. First, we assessed the associations between possible demographic factors and risk recognition following stratification by age group ( $<60$ years, $\geq 60$ years). Second, the age group $(1,<50$ years; 2, 50-59 years; 3, 60-69 years; and 4: $\geq 70$ years) was additionally adjusted as a covariate in logistic regression models. The $\mathrm{p}$ values of $<0.05$ were defined as significant when they were set to two-sided. Statistical analyses were performed using IBM SPSS version 23.0 (IBM Corp., Armonk, NY, USA) and SAS version 9.4 (SAS Institute, Cary, NC, USA). Spearman correlation was visualized by using the package corrplot (R 3.5.3; R Foundation for Statistical Computing, Vienna, Austria).

\section{Results}

\section{Study populations}

The mean age of the participating CKD patients $(\mathrm{n}=306)$ was $61.0 \pm 12.5$ years, and 209 patients $(68.3 \%)$ were male (Table 1). The mean serum creatinine and eGFR of the patients were $1.8 \pm 0.7 \mathrm{mg} / \mathrm{dL}$ and $43.2 \pm 20.7 \mathrm{~mL} / \mathrm{min} / 1.73$ $\mathrm{m}^{2}$, respectively. A total of 187 patients $(61.1 \%)$ showed an eGFR less than $45 \mathrm{~mL} / \mathrm{min} / 1.73 \mathrm{~m}^{2}$ and were regarded as the advanced CKD group. Advanced CKD was found more frequently among older and female patients; these patients tended to show lower serum hemoglobin, platelet, serum albumin, bilirubin, and a higher proportion of overt proteinuria (Table 1).

The majority of patients had completed a high school education (35.9\%) or higher (29.2\%). A total of 137 participants (44.8\%) were working either full or part-time. There were 56 
Table 1. Demographic and clinical characteristics of CKD patients in the SKETCH cohort

\begin{tabular}{|c|c|c|c|c|}
\hline Variable & Total population $(n=306)$ & Early CKD $(n=119)$ & Advanced CKD ( $\mathrm{n}=187)$ & $p$-value \\
\hline Age (yr) & $61.0 \pm 12.5$ & $58.6 \pm 13.2$ & $62.6 \pm 11.8$ & 0.007 \\
\hline Male sex & $209(68.3)$ & $93(78.2)$ & $116(62.0)$ & 0.003 \\
\hline Height (cm) & $164.7 \pm 9.2$ & $166.8 \pm 9.5$ & $163.3 \pm 8.7$ & 0.001 \\
\hline Body weight (kg) & $69.5 \pm 13.1$ & $72.1 \pm 13.6$ & $67.8 \pm 12.6$ & 0.005 \\
\hline Body mass index $\left(\mathrm{kg} / \mathrm{m}^{2}\right)$ & $25.6 \pm 4.2$ & $25.9 \pm 4.8$ & $25.3 \pm 3.7$ & 0.22 \\
\hline SBP (mmHg) & $128.1 \pm 14.8$ & $127.7 \pm 13.9$ & $128.4 \pm 15.3$ & 0.71 \\
\hline $\mathrm{DBP}(\mathrm{mmHg})$ & $73.3 \pm 12.2$ & $75.5 \pm 10.2$ & $72.0 \pm 13.2$ & 0.008 \\
\hline Diabetes mellitus & $166(54.2)$ & $57(47.9)$ & $109(58.3)$ & 0.08 \\
\hline WBC $\left(\times 10^{3} / \mu \mathrm{L}\right)$ & $7.0 \pm 1.9$ & $6.8 \pm 1.8$ & $7.1 \pm 2.0$ & 0.16 \\
\hline Hemoglobin (g/dL) & $12.6 \pm 2.0$ & $13.9 \pm 1.6$ & $11.8 \pm 1.8$ & $<0.001$ \\
\hline Platelets $\left(\times 10^{3} / \mu \mathrm{L}\right)$ & $223.0 \pm 62.6$ & $234.3 \pm 58.4$ & $215.9 \pm 64.2$ & 0.01 \\
\hline Glucose (mg/dL) & $120.1 \pm 44.0$ & $118.4 \pm 36.6$ & $121.1 \pm 48.2$ & 0.58 \\
\hline Total cholesterol (mg/dL) & $158.3 \pm 53.7$ & $159.4 \pm 40.4$ & $157.5 \pm 60.9$ & 0.77 \\
\hline Protein $(\mathrm{g} / \mathrm{dL})$ & $7.0 \pm 0.5$ & $7.1 \pm 0.6$ & $6.9 \pm 0.5$ & 0.02 \\
\hline Albumin (g/dL) & $4.3 \pm 0.4$ & $4.5 \pm 0.4$ & $4.3 \pm 0.4$ & $<0.001$ \\
\hline Bilirubin (mg/dL) & $0.5 \pm 0.3$ & $0.6 \pm 0.3$ & $0.5 \pm 0.3$ & $<0.001$ \\
\hline Uric acid (mg/dL) & $6.5 \pm 1.8$ & $6.3 \pm 1.7$ & $6.6 \pm 1.8$ & 0.20 \\
\hline BUN (mg/dL) & $28.8 \pm 12.8$ & $20.1 \pm 6.5$ & $34.3 \pm 12.7$ & $<0.001$ \\
\hline Creatinine $(\mathrm{mg} / \mathrm{dL})$ & $1.8 \pm 0.7$ & $1.2 \pm 0.3$ & $2.2 \pm 0.6$ & $<0.001$ \\
\hline GFR (CKD-EPI) (mL/min/1.73 m²) & $43.2 \pm 20.7$ & $63.2 \pm 18.4$ & $30.4 \pm 8.1$ & $<0.001$ \\
\hline uPCR (g/gCr) & $0.9 \pm 1.3$ & $0.5 \pm 0.8$ & $1.1 \pm 1.6$ & $<0.001$ \\
\hline
\end{tabular}

Data are expressed as mean \pm standard deviation or number (\%).

SKETCH cohort, the Study on Kidney Disease and Environmental Chemicals (Clinical Trial No. NCT04679168).

BUN, blood urea nitrogen; CKD, chronic kidney disease; CKD-EPI, Chronic Kidney Disease Epidemiology Collaboration equation; DBP, diastolic blood pressure; GFR, glomerular filtration rate; SBP, systolic blood pressure; UPCR, urine protein creatinine ratio; WBC, white blood cells.

(18.3\%) and 85 participants $(27.8 \%)$ with current smoking and alcohol consumption, respectively. Most participating CKD patients stated their health status as 'not good' (43.1\%) or 'bad' (46.7\%).

\section{Risk perception}

At the time of the survey during the global pandemic of COVID-19, in general, most participating patients (89\%) perceived the risk of infection as 'serious.' More people considered the general risk of infection nationwide (i.e., 'nationwide in general') as serious (76.1\%) than the risk for their city of residence (55.4\%) or the individual (45.6\%) (Supplementary Fig. 2, available online).

In CKD patients, risk perception for the city of residence or the individual was also significantly correlated with trends of COVID-19 case reporting (Supplementary Table 1, available online). Based on this result, we used the risk perception variable as the risk on the individuals to evaluate the impact on behavior changes. The level of perceived risk in general populations appeared to be related to the trends in cases of COVID-19 infection (Fig. 1A). Most patients of the cohort were enrolled after the first peak of the pandemic. Compared to the general population of Korea $(n=1,000)$, CKD patients showed much greater levels of the perceived risk from COVID-19 infection (Fig. 1B).

Older patients with lower education and income were more likely to perceive a greater risk of infection. The stage of CKD, however, did not influence the risk perception (Table 2). When stratified by age group, the associations of education and income with risk perception disappeared (Supplementary Table 2, available online).

\section{Behavioral changes during the COVID-19 pandemic}

The frequencies of 'public transport use' and 'public place visit' were significantly decreased in all populations regardless of the frequency of such behaviors before the pandemic 

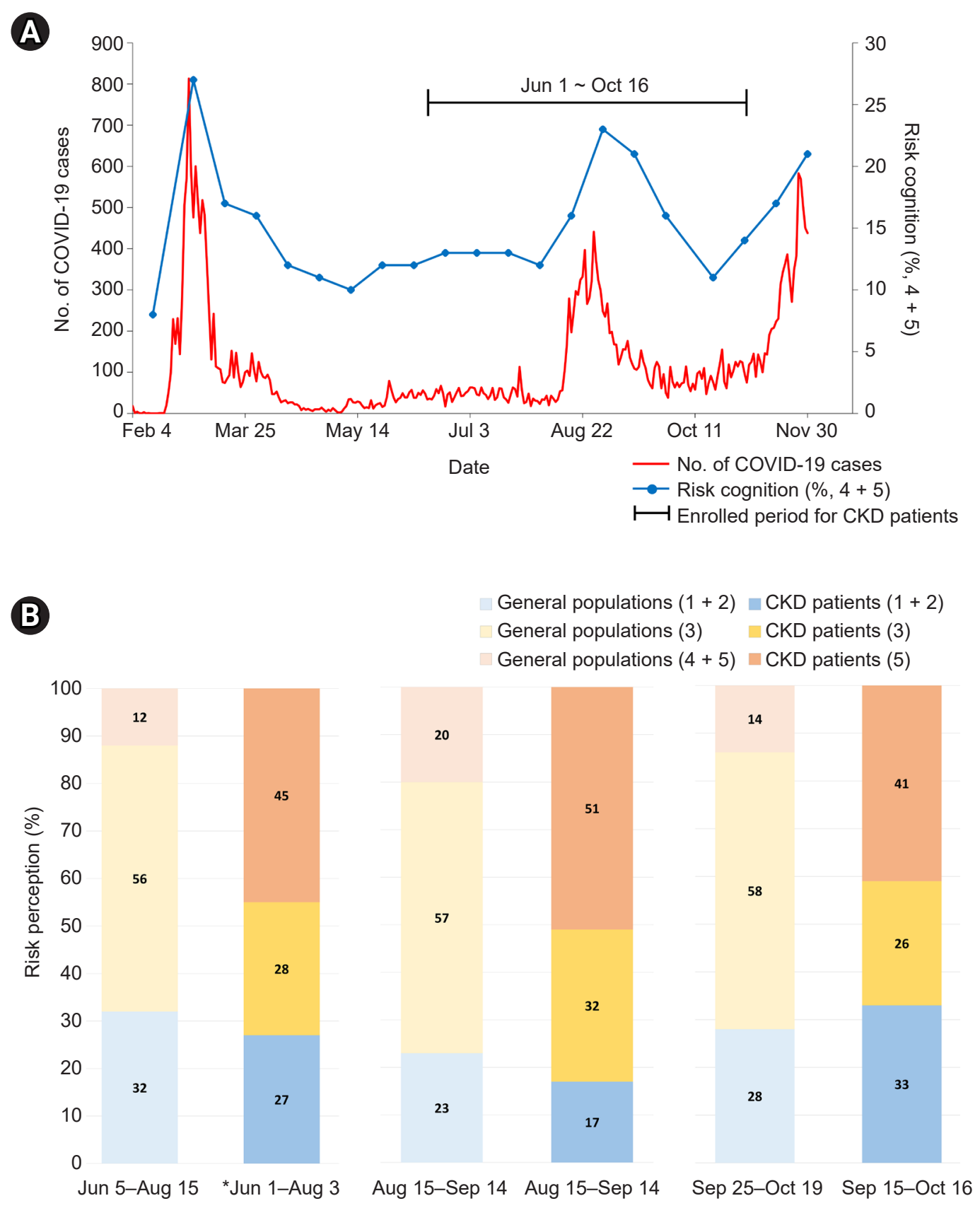

Figure 1. Distribution of enrolled participants and risk cognition according to the pandemic. (A) Number of participating chronic kidney disease patients recruited in the Study on Kidney Disease and Environmental Chemicals (SKETCH, Clinical Trial No. NCTO4679168) cohort in comparison with the daily number of confirmed cases of coronavirus disease 2019 (COVID-19) nationwide (left Y-axis and red line). The proportion of people who answered 'serious' or 'very serious' for the possibility of infection by COVID-19 in the general populations is shown by the blue dotted line (right Y-axis). (B) Comparison of risk perception between the general population of Korea $(n=1,000$, left) and those who were participating in the SKETCH cohort (right) for given periods. Specific time periods were demonstrated under the graph, and "*” represented the period for chronic kidney disease (CKD) patients. Crimson red, gray, and blue colors indicate the proportion of the population who answered 'high' ('4' or '5'), 'moderate' ('3'), and 'low' ('1' or '2') levels of risk perception, respectively.

*The statistics for the incidence of COVID-19 represented with a black line were obtained from the Korean Statistical Information Service. The risk perception for COVID-19 in the general population was obtained from Hankook Research (https://hrcopinion.co.kr/ covid-19, accessed December 18, 2020), which collected data from 1,000 subjects through biweekly survey events. 
Table 2. Perceived risk of COVID-19 infection by demographic and socioeconomic characteristics, and CKD stage

\begin{tabular}{|c|c|c|}
\hline \multirow{2}{*}{ Variable } & \multicolumn{2}{|c|}{ Total $(n=306)$} \\
\hline & OR $(95 \% \mathrm{Cl})$ & p-value \\
\hline \multicolumn{3}{|l|}{ Age (yr) (Ref, <50) } \\
\hline $50 s$ & $1.49(0.69-3.20)$ & 0.31 \\
\hline $60 \mathrm{~s}$ & $3.26(1.59-6.67)$ & $0.001 *$ \\
\hline$\geq 70$ & $3.92(1.89-8.15)$ & $<0.001 *$ \\
\hline \multicolumn{3}{|l|}{ Sex (Ref, male) } \\
\hline Female & $1.06(0.65-1.72)$ & 0.82 \\
\hline \multicolumn{3}{|c|}{ Educationa (Ref, selementary school) } \\
\hline Middle school & $0.62(0.29-1.35)$ & 0.23 \\
\hline High school & $0.44(0.23-0.85)$ & $0.01 *$ \\
\hline$\geq$ College & $0.32(0.16-0.64)$ & $0.001 *$ \\
\hline \multicolumn{3}{|c|}{ Incomea (Ref, <\$1,000) } \\
\hline $1,000-2,000$ & $1.21(0.62-2.36)$ & 0.58 \\
\hline $2,000-3,000$ & $0.89(0.42-1.87)$ & 0.75 \\
\hline $3,000-5,000$ & $0.44(0.23-0.87)$ & $0.02 *$ \\
\hline$\geq 5,000$ & $0.27(0.13-0.55)$ & $<0.001 *$ \\
\hline \multicolumn{3}{|l|}{$\mathrm{CCl}($ Ref, <4) } \\
\hline$\geq 4$ & $1.66(1.05-2.62)$ & $0.03 *$ \\
\hline Diabetes & $1.35(0.86-2.12)$ & 0.20 \\
\hline \multicolumn{3}{|c|}{ CKD stage (Ref, early CKD) } \\
\hline Advanced CKD & $1.46(0.91-2.33)$ & 0.11 \\
\hline
\end{tabular}

$\mathrm{CCl}$, Charlson comorbidity index; $\mathrm{Cl}$, confidence interval; Ref, reference; CKD, chronic kidney disease; COVID-19, coronavirus disease 2019; OR, odds ratio.

${ }^{a} n=305$ (total) and $n=176$ ( $\geq 60$ years).

* $p<0.05$.

(Table 3). The frequency of 'private vehicle use' tended to increase among those who seldom used private transport before the pandemic, but among those who frequently used private vehicles, the frequency of use decreased (Supplementary Fig. 3A, available online).

During the pandemic, CKD patients showed significant increases in several hygiene-related behaviors. The frequency of handwashing, showering, face mask use, and hand sanitizer use was significantly increased during the COVID-19 pandemic. In particular, there were no subjects who did not wear a face mask during the pandemic ( $\mathrm{p}<$ $0.001)$. In addition, the frequencies of laundry and house cleaning were significantly increased $(\mathrm{p}<0.001)$ (Supplementary Fig. 3B).

Regarding the frequency of exercise, i.e., physical activity over 30 minutes, no change was observed in general ( $\mathrm{n}=$ $306)$ and in those who exercised on an irregular basis $(\mathrm{n}=$
128). Among those who exercised regularly $(\mathrm{n}=178)$ or in gyms $(n=28)$, however, the frequency of exercise significantly decreased.

\section{Factors associated with social distancing behaviors}

Several demographic and socioeconomic factors were associated with behaviors related to social distancing (Table 4 ). Younger patients ( $<50$ years old) showed less frequent public place visits and more exercise. Patients with higher income showed $>1.5$ ORs for less frequent public place visits and longer stays-at-home (Supplementary Table 3, available online). The positive associations of stay-at-home with sex and income became stronger after age adjustment (Table 4).

Higher risk perception was significantly associated with decreased use of public transport (OR, 2.00; 95\% CI, 1.09-3.66), and the association remained significant after adjustment for age (adjusted OR, 1.91; 95\% CI, 1.01-3.60) (Table 4). Among patients with diabetes, the negative association with the frequency of public place visits was attenuated by age adjustment (adjusted OR, 0.66; 95\% CI, 0.41-1.05). In addition, advanced CKD patients tended to stay-at-home for shorter times even after adjusting for age (adjusted OR, 0.54; 95\% CI, 0.33-0.88) (Table 4).

\section{Factors associated with hygiene-related behaviors}

Behaviors related to personal hygiene were related to age, education, and CKD stage, even though clear linearities were often unseen (Supplementary Table 4, available online). Hand sanitizer use was more frequent among people with higher education and higher income (Table 5). In addition, patients with higher income tended to perform laundry and house cleaning more frequently. The positive association between income and frequency of laundry remained significant after age adjustment (Table 5). The frequencies of handwashing and face mask use, however, were not influenced by any factors assessed in the present study.

The level of risk perception was not related to hygiene-related behaviors. In addition, CCI status was not associated with hygienic behaviors except for hand sanitizer use (Table 5). However, among patients with diabetes, the use of hand sanitizer was significantly decreased (OR, 0.47; 95\% CI, 0.23-0.99), and the number of clothes used between 
Table 3. Behavioral changes related to social distancing, hygiene, and exercise during COVID-19 pandemic compared with before the pandemic

\begin{tabular}{|c|c|c|c|c|c|c|}
\hline Factor & Category & No & Before & After & $\%$ increase & $\%$ decrease \\
\hline \multirow[t]{4}{*}{ Social distancing } & Public places (time/wk) & 306 & $2.3 \pm 2.7$ & $1.1 \pm 2.2 * * *$ & 1.6 & 42.8 \\
\hline & Public transport (time/wk) & 306 & $1.9 \pm 3.2$ & $1.4 \pm 3.1 * * *$ & 2.6 & 17.0 \\
\hline & Personal transport (time/wk) & 304 & $1.1 \pm 2.0$ & $1.1 \pm 2.0$ & 6.9 & 5.3 \\
\hline & Stay-at-home (hr/day) & 306 & $12.0 \pm 5.7$ & $13.5 \pm 5.7 * * *$ & 34.0 & 1.6 \\
\hline \multirow[t]{7}{*}{ Hygiene } & Mask (time/wk) & 306 & $1.0 \pm 2.2$ & $6.2 \pm 1.7 * * *$ & 85.0 & 0.3 \\
\hline & Hand sanitizer (time/wk) & 306 & $1.4 \pm 4.9$ & $8.4 \pm 9.4 * * *$ & 87.3 & 0.3 \\
\hline & Clothes (time before laundry) & 306 & $1.9 \pm 1.1$ & $2.0 \pm 1.4$ & 15.0 & 11.1 \\
\hline & Laundry (time/wk) & 306 & $3.3 \pm 2.1$ & $3.7 \pm 2.1 * * *$ & 23.5 & 0.7 \\
\hline & Cleaning the house (time/wk) & 306 & $4.6 \pm 2.5$ & $5.0 \pm 2.6 * * *$ & 22.5 & 1.3 \\
\hline & Soap (time/day) & 306 & $3.3 \pm 3.6$ & $6.1 \pm 5.0 * * *$ & 80.4 & 1.3 \\
\hline & Shower (time/day) & 306 & $1.1 \pm 0.7$ & $1.3 \pm 0.8 * * *$ & 19.9 & 0.3 \\
\hline \multirow[t]{10}{*}{ Exercise } & Total exercise (time/wk) & 306 & $2.7 \pm 2.8$ & $2.5 \pm 2.7$ & 12.4 & 14.7 \\
\hline & Irregular exercise (time/wk) & 128 & $0.7 \pm 1.3$ & $0.9 \pm 1.5$ & 15.6 & 7.0 \\
\hline & Regular exercise (time/wk) & 178 & $4.1 \pm 2.7$ & $3.7 \pm 2.8 * *$ & 10.1 & 20.2 \\
\hline & Exercise at gym (time/wk) & 28 & $3.6 \pm 1.9$ & $2.5 \pm 2.1 *$ & 14.3 & 42.9 \\
\hline & Without diabetes (time/wk) & 140 & $2.4 \pm 2.4$ & $2.1 \pm 2.3 *$ & 9.3 & 17.1 \\
\hline & With diabetes (time/wk) & 166 & $2.9 \pm 3.1$ & $2.9 \pm 3.0$ & 15.1 & 12.7 \\
\hline & $\mathrm{CCl}<4$ (time/wk) & 142 & $2.4 \pm 2.4$ & $2.0 \pm 2.2^{*}$ & 9.2 & 19.0 \\
\hline & $\mathrm{CCl} \geq 4$ (time/wk) & 164 & $3.0 \pm 3.1$ & $3.0 \pm 3.0$ & 14.0 & 9.8 \\
\hline & Early CKD (time/wk) & 116 & $2.6 \pm 2.6$ & $2.4 \pm 2.4$ & 13.8 & 18.1 \\
\hline & Advanced CKD (time/wk) & 190 & $2.7 \pm 2.9$ & $2.6 \pm 2.9$ & 11.6 & 12.6 \\
\hline
\end{tabular}

Data are expressed as number only, mean \pm standard deviation, or percentage only.

CCI, Charlson comorbidity index; CKD, chronic kidney disease; COVID-19, coronavirus disease 2019.

Wilcoxon signed-rank test was conducted; ${ }^{*} p<0.05, * * p<0.01, * * * p<0.001$.

laundering was significantly increased (OR, 0.46; 95\% CI, 0.21-0.97) compared to non-diabetes patients even after adjustment for age (Table 5). Advanced CKD status showed a significant association only with the decreased frequency of showering (OR, 0.52; 95\% CI, 0.29-0.93).

\section{Factors associated with physical activities}

In general, exercise behavior among CKD patients was not affected during the COVID-19 pandemic. Among those who had exercised regularly, the frequency of exercise was negatively associated with age (OR, 0.26; 95\% CI, 0.09$0.74)$. Among those who took regular exercise, education (OR, 5.47; 95\% CI, 1.22-24.62) and income (OR, 6.59; 95\% CI, 1.84-23.68) status were positively associated with exercise (Supplementary Table 5, available online). The positive association of income remained significant even after adjustment for age in patients who took regular exercise (Table 6).

\section{Discussion}

Among the participating patients with CKD, the COVID-19 pandemic significantly altered behavioral characteristics related to social distancing, hygiene, and physical activity. Since the outbreak of the COVID-19 pandemic, several observations on behavioral changes among chronic disease patients have been reported [15,16]; however, no reports have been made on CKD patients. Because such behavioral changes may influence the progress or prognosis of underlying diseases, including CKD and also COVID-19 infection, characterizing behavioral changes in patients has important clinical implications $[5,6,8]$.

The participating patients with CKD showed a greater level of risk perception toward COVID-19 infection, i.e., >2 times greater proportion of the SKETCH cohort answered 'serious' or 'very serious' for the possibility of COVID-19 infection risk, compared to the general population of Korea. Several hygienic behaviors were also more frequently 
Table 4. Behavioral changes related to social distancing by demographic and socioeconomic characteristics and comorbidities after age adjustment

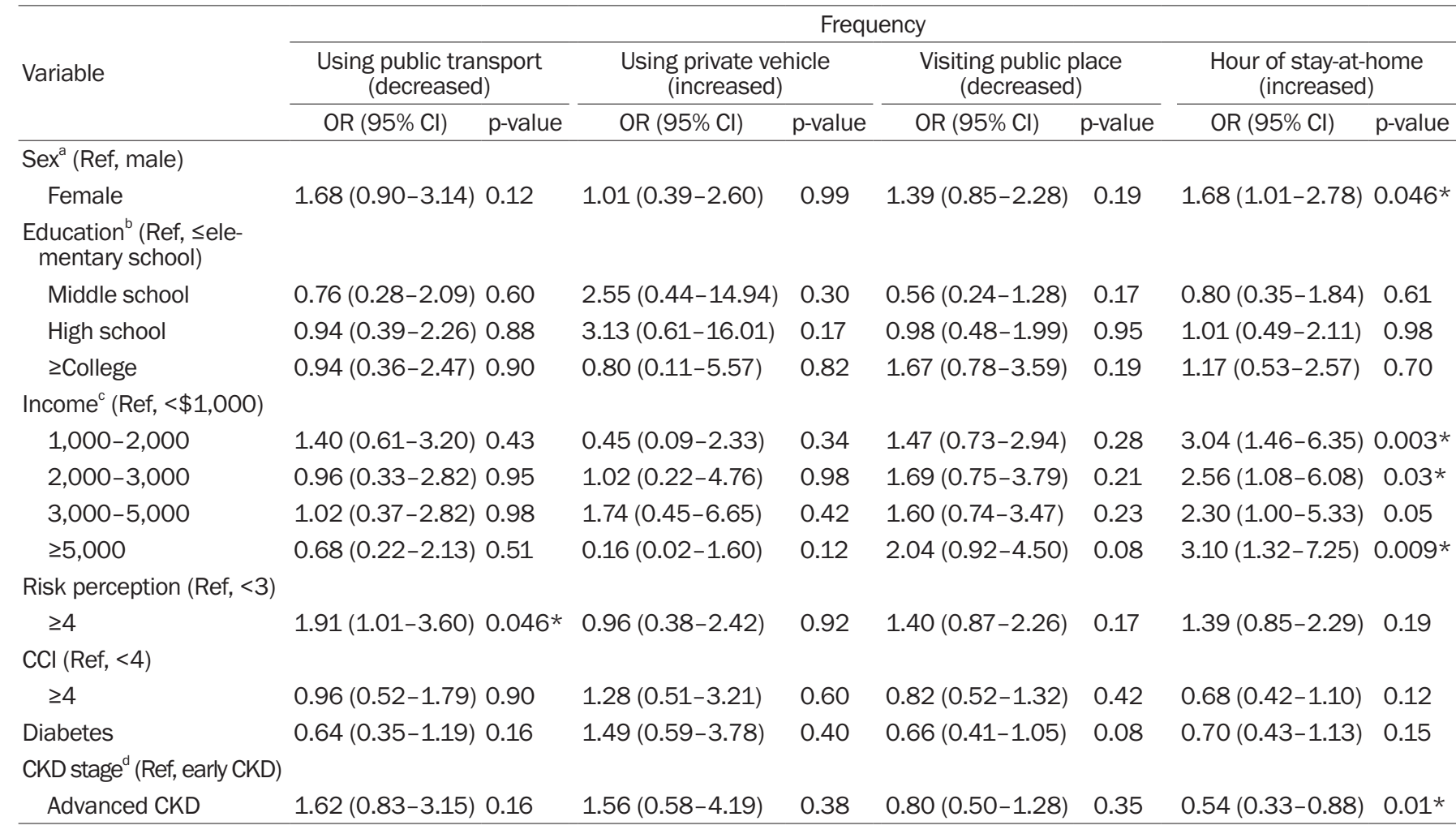

$\mathrm{CCl}$, Charlson comorbidity index; $\mathrm{Cl}$, confidence interval; $\mathrm{CKD}$, chronic kidney disease; OR, odds ratio; Ref, reference.

$\mathrm{a}, \mathrm{d}=304$ (personal transport). ${ }^{\mathrm{b}, \mathrm{c}} \mathrm{n}=303$ (personal transport).

$* p<0.05$.

adopted among CKD patients than the general Korean population, for example, the use of face masks ( $100 \%$ vs. $63.2 \%$ in the general population) and hand hygiene (93.7\% vs. $67.8 \%$ in the general population) during the first wave of the pandemic (February 2020) [17]. Risk perception is generally considered to be related to culture, worldview, experience, and prosocial values and is often amplified through friends and family [18]. The higher perceived risk of infection among the participating CKD patients may be partly due to the underlying disease; comorbidities were suggested as one key indicator that could increase the perception of risk [19].

Socioeconomic status including education, income (higher), and sex (female) are negatively correlated with unhealthy behaviors $[20,21]$. In addition, the severity of comorbidities is closely related to the tendency toward healthy behavior. Patients with more comorbidities tend to engage in more conservative behaviors for the sake of their health [22]. In United States adults $(n=6,463)$ from March to April
2020, hygiene-related behaviors such as handwashing and surface disinfection were more common among females and those older patients with higher income, higher education, and self-rated good health [21]. Except for self-rated health status, these findings are generally comparable to the observations made for the present CKD patients. Longer stay-at-home behavior among CKD patients may reflect higher levels of risk perception among these patients in comparison to the general population of Korea (Fig. 1B). Although the stage of CKD was inversely associated with increased stay-at-home time, it might be related to a discrepancy between risk perception and behavior change due to the worse socioeconomic and education status in advanced CKD patients.

The lack of significant changes in the frequencies of handwashing and face mask use by key socioeconomic factors such as education and income warrant further discussion because these factors have been recognized as drivers of healthy or conservative behaviors. Null associations with 


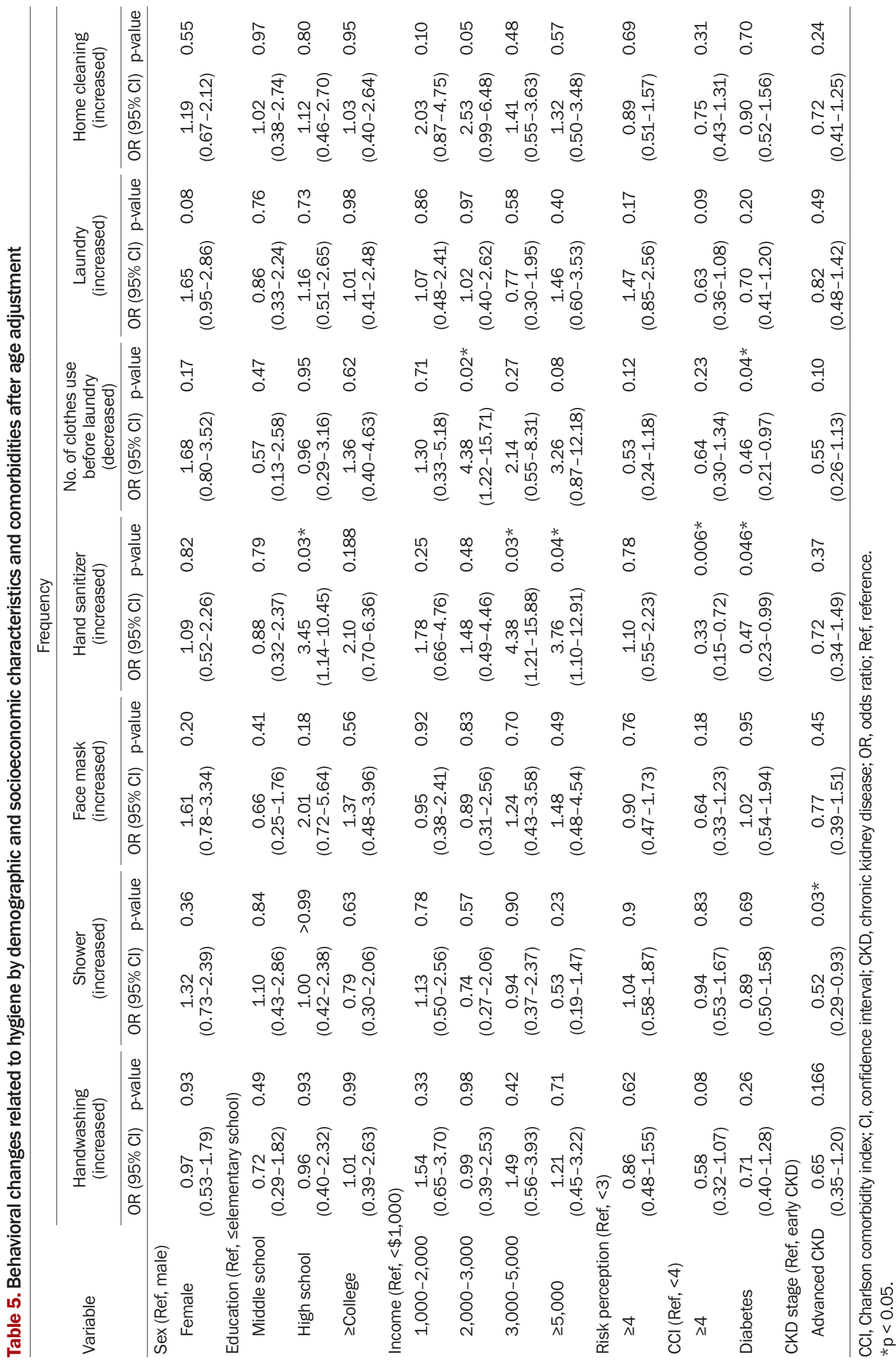


Table 6. Behavioral changes related to exercise by demographic and socioeconomic characteristics and comorbidities after age adjustment

\begin{tabular}{|c|c|c|c|c|c|c|}
\hline \multirow{3}{*}{ Variable } & \multicolumn{6}{|c|}{ Increased frequency } \\
\hline & \multicolumn{2}{|l|}{ Overall } & \multicolumn{2}{|c|}{ Irregular exercise $(n=128)$} & \multicolumn{2}{|c|}{ Regular exercise $(n=178)$} \\
\hline & OR $(95 \% \mathrm{Cl})$ & p-value & OR $(95 \% \mathrm{Cl})$ & p-value & OR (95\% Cl) & p-value \\
\hline \multicolumn{7}{|l|}{ Sex (Ref, male) } \\
\hline Female & $0.63(0.29-1.40)$ & 0.26 & $0.65(0.22-1.93)$ & 0.44 & $0.66(0.20-2.16)$ & 0.496 \\
\hline \multicolumn{7}{|c|}{ Education (Ref, smiddle school) } \\
\hline 2High school & $1.51(0.62-3.66)$ & 0.37 & $0.88(0.29-2.67)$ & 0.82 & $3.41(0.66-17.73)$ & 0.15 \\
\hline \multicolumn{7}{|c|}{ Income (Ref, <\$2,000) } \\
\hline$\geq \$ 2,000$ & $1.72(0.77-3.85)$ & 0.19 & $0.78(0.27-2.28)$ & 0.65 & $4.76(1.18-19.21)$ & $0.03 *$ \\
\hline \multicolumn{7}{|c|}{ Risk perception (Ref, <3) } \\
\hline$\geq 4$ & $0.58(0.27-1.21)$ & 0.15 & $1.01(0.37-2.75)$ & 0.99 & $0.32(0.10-1.05)$ & 0.06 \\
\hline \multicolumn{7}{|l|}{ CCl (Ref, <4) } \\
\hline$\geq 4$ & $2.10(1.01-4.38)$ & $0.048 *$ & $3.31(1.09-10.00)$ & $0.03 *$ & $1.35(0.49-3.75)$ & 0.56 \\
\hline Diabetes & $1.96(0.95-4.06)$ & 0.07 & $2.61(0.92-7.38)$ & 0.07 & $1.51(0.54-4.25)$ & 0.43 \\
\hline \multicolumn{7}{|l|}{ CKD stage } \\
\hline Advanced CKD & $0.87(0.43-1.74)$ & 0.69 & $1.30(0.47-3.57)$ & 0.61 & $0.53(0.19-1.43)$ & 0.21 \\
\hline
\end{tabular}

Due to no observations for explanatory variable (frequency of exercise) in some age, education, household income groups, the groups were redefined. $\mathrm{CCl}$, Charlson comorbidity index; $\mathrm{Cl}$, confidence interval; $\mathrm{CKD}$, chronic kidney disease; OR, odds ratio; Ref, reference. $* \mathrm{p}<0.05$.

handwashing and face mask use remained the same regardless of age adjustment (Table 5). These observations can be explained by the almost unanimous use of face masks $(100 \%)$ and much greater frequency of handwashing $(86.3 \%$ vs. $67.8 \%$ among the general population [17]) in the present CKD patients regardless of socioeconomic status or sex.

Stringent social distancing or self-isolation may produce side effects such as physical inactivity, which may lead to other health issues [23]. In the United Kingdom, the prevalence of physical activity was found to be substantially lower in general among adults than before the pandemic, and this change was attributed to the requirement for self-isolation and stay-at-home orders [24]. Moreover, patients with comorbidities tended to be less physically active during the pandemic than the matching healthy population $[25,26]$. Unlike previous studies that reported notable decreases in physical activity, our findings showed that the physical activity of CKD patients in Korea was generally unaffected during the COVID-19 pandemic (Supplementary Fig. 3C). These observations indicate the target population to whom clinical recommendations for exercise should be delivered.

Physical activity is an important component in the management of kidney disease; it helps to reduce inflammation, improve glomerular filtration rate, and reduce albuminuria
[12-14] and is often related to the quality of life in CKD patients [27]. Guidelines for adequate levels of exercise are therefore warranted for CKD patients, especially during the pandemic, which has led to reduced physical activities. One survey of patients with kidney transplantation in Germany showed that a telemedicine-based aftercare program could efficiently increase overall activity during the COVID-19 pandemic, although sports activities decreased [28]. This interesting observation may be attributable not only to an effective follow-up care program but also to the less strict social distancing policy of Germany that allowed outdoor physical activity [28]. Follow-up and intervention programs designed specifically for CKD patients to maintain or enhance physical activity are recommended for this vulnerable population during the pandemic when self-isolation is involved.

This study is the first report of the behavioral characteristics and changes among CKD patients during the COVID-19 pandemic in Korea. As a cross-sectional study of a group of CKD patients recruited via convenience sampling, the observations of this study may not be generalizable. In addition, there are limitations for exact comparison to general populations because of the restriction on the population with CKD. Finally, because of the structural limitations for 
reflecting the different baseline characteristics between the subgroups in the logistic regression analysis, ordinary concepts were not applicable in some of the results. Despite presenting only a snapshot observation on CKD patients during this global pandemic, the results of this study can help health practitioners design relevant recommendations for CKD patients not only to reduce the likelihood of COVID-19 infection but also to maintain health-related behaviors.

\section{Conflicts of interest}

All authors have no conflicts of interest to declare.

\section{Funding}

This work was supported by Seoul National University Research Grant in 2020 (SRnD 800-20200496).

\section{Acknowledgments}

The authors thank the participating patients and clinical research coordinators for their contribution.

\section{Authors' contributions}

Conceptualization: YK, IL, KC, JPL

Investigation: YK, JL, JYP, JNA, KDY, YCK, WYP, KJ, DKK

Data curation and Formal analysis: IL, YK, MY

Funding acquisition: JPL

Project administration: KC, JPL

Writing-original draft: $\mathrm{YK}$

Writing-review \& editing: All authors

All authors read and approved the final manuscript.

\section{ORCID}

Yaerim Kim, https://orcid.org/0000-0003-1596-1528

Inae Lee, https://orcid.org/0000-0002-9946-8652

Jeonghwan Lee, https://orcid.org/0000-0003-3199-635X

Jae Yoon Park, https://orcid.org/0000-0001-8986-7492

Jung Nam An, https://orcid.org/0000-0001-5108-1005

Kyung Don Yoo, https://orcid.org/0000-0001-6545-6517

Yong Chul Kim, https://orcid.org/0000-0003-3215-8681

Woo Yeong Park, https://orcid.org/0000-0003-2662-2898
Kyubok Jin, https://orcid.org/0000-0002-7836-8863

Younglim Kho, https://orcid.org/0000-0002-2590-4722

Myoungsoon You, https://orcid.org/0000-0001-9869-014X

Dong Ki Kim, https://orcid.org/0000-0002-5195-7852

Kyungho Choi, https://orcid.org/0000-0001-7460-792X

Jung Pyo Lee, https://orcid.org/0000-0002-4714-1260

\section{References}

1. Lee D, Heo K, Seo Y, et al. Flattening the curve on COVID-19: South Korea's measures in tackling initial outbreak of coronavirus. Am J Epidemiol 2021;190:496-505.

2. Majeed A, Seo Y, Heo K, Lee D. Can the UK emulate the South Korean approach to COVID-19? BMJ 2020;369:m2084.

3. Park HC, Kim DH, Yoo KD, et al. Korean clinical practice guidelines for preventing transmission of coronavirus disease 2019 (COVID-19) in hemodialysis facilities. Kidney Res Clin Pract 2020;39:145-150.

4. GBD Chronic Kidney Disease Collaboration. Global, regional, and national burden of chronic kidney disease, 1990-2017: a systematic analysis for the Global Burden of Disease Study 2017. Lancet 2020;395:709-733.

5. Dalrymple LS, Go AS. Epidemiology of acute infections among patients with chronic kidney disease. Clin J Am Soc Nephrol 2008;3:1487-1493.

6. Wang HE, Gamboa C, Warnock DG, Muntner P. Chronic kidney disease and risk of death from infection. Am J Nephrol 2011;34:330-336.

7. Jin DC. Analysis of mortality risk from Korean hemodialysis registry data 2017. Kidney Res Clin Pract 2019;38:169-175.

8. Gansevoort RT, Hilbrands LB. CKD is a key risk factor for COVID-19 mortality. Nat Rev Nephrol 2020;16:705-706.

9. Hall G, Laddu DR, Phillips SA, Lavie CJ, Arena R. A tale of two pandemics: How will COVID-19 and global trends in physical inactivity and sedentary behavior affect one another? Prog Cardiovasc Dis 2021;64:108-110.

10. Knell G, Robertson MC, Dooley EE, Burford K, Mendez KS. Health behavior changes during COVID-19 pandemic and subsequent "stay-at-home" orders. Int J Environ Res Public Health 2020;17:6268.

11. World Health Organization (WHO). Physical inactivity: a global public health problem [Internet]. Geneva: WHO; 2020 [cited 2021 Mar 16]. Available from: https://www.who.int/ncds/prevention/physical-activity/inactivity-global-health-problem/ en/. 
12. Barcellos FC, Santos IS, Umpierre D, Bohlke M, Hallal PC. Effects of exercise in the whole spectrum of chronic kidney disease: a systematic review. Clin Kidney J 2015;8:753-765.

13. Hellberg M, Höglund P, Svensson P, Clyne N. Randomized controlled trial of exercise in CKD: the RENEXC Study. Kidney Int Rep 2019;4:963-976.

14. Pei G, Tang Y, Tan L, Tan J, Ge L, Qin W. Aerobic exercise in adults with chronic kidney disease (CKD): a meta-analysis. Int Urol Nephrol 2019;51:1787-1795.

15. Wolf MS, Serper M, Opsasnick L, et al. Awareness, attitudes, and actions related to COVID-19 among adults with chronic conditions at the onset of the U.S. outbreak: a cross-sectional survey. Ann Intern Med 2020;173:100-109.

16. Huynh G, Nguyen MQ, Tran TT, et al. Knowledge, attitude, and practices regarding COVID-19 among chronic illness patients at outpatient departments in Ho Chi Minh City, Vietnam. Risk Manag Healthc Policy 2020;13:1571-1578.

17. Lee M, You M. Psychological and behavioral responses in South Korea during the early stages of coronavirus disease 2019 (COVID-19). Int J Environ Res Public Health 2020;17:2977.

18. Dryhurst S, Schneider CR, Kerr J, et al. Risk perceptions of COVID-19 around the world. J Risk Res 2020;23:994-1006.

19. He S, Chen S, Kong L, Liu W. Analysis of risk perceptions and related factors concerning COVID-19 epidemic in Chongqing, China. J Community Health 2021;46:278-285.

20. Pampel FC, Krueger PM, Denney JT. Socioeconomic disparities in health behaviors. Annu Rev Sociol 2010;36:349-370.

21. Brown LG, Hoover ER, Barrett CE, Vanden Esschert KL, Collier SA, Garcia-Williams AG. Handwashing and disinfection precau- tions taken by U.S. adults to prevent coronavirus disease 2019, Spring 2020. BMC Res Notes 2020;13:550.

22. Nothwehr F, Perkins AJ. Relationships between comorbidity and health behaviors related to hypertension in NHANES III. Prev Med 2002;34:66-71.

23. Füzéki E, Groneberg DA, Banzer W. Physical activity during COVID-19 induced lockdown: recommendations. J Occup Med Toxicol 2020;15:25.

24. Spence JC, Rhodes RE, McCurdy A, Mangan A, Hopkins D, Mummery WK. Determinants of physical activity among adults in the United Kingdom during the COVID-19 pandemic: the DUK-COVID study. Br J Health Psychol 2021;26:588-605.

25. Dobler CL, Krüger B, Strahler J, Weyh C, et al. Physical activity and mental health of patients with pulmonary hypertension during the COVID-19 pandemic. J Clin Med 2020;9:4023.

26. Rogers NT, Waterlow NR, Brindle H, et al. Behavioral change towards reduced intensity physical activity is disproportionately prevalent among adults with serious health issues or self-perception of high risk during the UK COVID-19 lockdown. Front Public Health 2020;8:575091.

27. Zelle DM, Klaassen G, van Adrichem E, Bakker SJ, Corpeleijn E, Navis G. Physical inactivity: a risk factor and target for intervention in renal care. Nat Rev Nephrol 2017;13:152-168.

28. Hanke AA, Sundermeier T, Boeck HT, et al. Influence of officially ordered restrictions during the first wave of COVID-19 pandemic on physical activity and quality of life in patients after kidney transplantation in a telemedicine based aftercare program: a KTx360 ${ }^{\circ}$ sub study. Int J Environ Res Public Health 2020;17:9144. 